\title{
On the metric dimension of strongly annihilating-ideal graphs of commutative rings
}

\author{
V. Soleymanivarniab \\ Department of Mathematics, \\ Science and Research Branch, \\ Islamic Azad University (IAU), \\ Tehran, Iran \\ email: soleymani.vali@yahoo.com
}

\author{
R. Nikandish* \\ Department of Mathematics, \\ Jundi-Shapur University of Technology, \\ Dezful, Iran \\ email: r.nikandish@ipm.ir
}

\author{
A. Tehranian \\ Department of Mathematics, \\ Science and Research Branch, \\ Islamic Azad University (IAU), Tehran, Iran \\ email: tehranian@srbiau.ac.ir
}

\begin{abstract}
Let $R$ be a commutative ring with identity and $A(R)$ be the set of ideals with non-zero annihilator. The strongly annihilatingideal graph of $R$ is defined as the graph $\operatorname{SAG}(R)$ with the vertex set $A(R)^{*}=A(R) \backslash\{0\}$ and two distinct vertices $I$ and $J$ are adjacent if and only if $\mathrm{I} \cap \operatorname{Ann}(J) \neq(0)$ and $J \cap \operatorname{Ann}(I) \neq(0)$. In this paper, we study the metric dimension of $\mathrm{SAG}(\mathrm{R})$ and some metric dimension formulae for strongly annihilating-ideal graphs are given.
\end{abstract}

2010 Mathematics Subject Classification: 13A99; 05C78; 05C12

Key words and phrases: metric dimension, strongly annihilating-ideal graph, commutative ring

${ }^{*}$ Corresponding author 


\section{Introduction}

The problem of finding the metric dimension of a graph was first studied by Harary and Melter [7]. Determining the metric dimension of a graph as an NP-complete problem has attracted many graph theorists and it has appeared in various applications of graph theory, for example pharmaceutical chemistry [5], robot navigation [8], combinatorial optimization [14] and so on. Recently, there was much work done in computing the metric dimension of graphs associated with algebraic structures. Calculating the metric dimension for the commuting graph of a dihedral group was done in [1], for the zero-divisor graphs of commutative rings in $[9,10,12]$, for the compressed zero-divisor graphs of commutative rings in [13], for total graphs of finite commutative rings in [6], for some graphs of modules in [11] and for annihilator graphs of commutative rings in [15]. Motivated by these papers, we study the metric dimension of another graph associated with a commutative ring.

Throughout this paper, all rings are assumed to be commutative with identity. The sets of all zero-divisors, nilpotent elements and maximal ideals are denoted by $Z(R), \operatorname{Nil}(R)$ and $\operatorname{Max}(R)$, respectively. For a subset $T$ of a ring $R$ we let $T^{*}=T \backslash\{0\}$. An ideal with non-zero annihilator is called an annihilatingideal. The set of annihilating-ideals of $R$ is denoted by $A(R)$. For every subset I of $R$, we denote the annihilator of I by $\operatorname{Ann}(\mathrm{I})$. A non-zero ideal I of $R$ is called essential if I has a non-zero intersection with every other non-zero ideal of $R$. The set of essential annihilating-ideal ideals of $R$ is denoted by $\operatorname{Ess}(R)$. The ring $R$ is said to be reduced if it has no non-zero nilpotent element. Some more definitions about commutative rings can be find in $[2,4]$.

We use the standard terminology of graphs following [18]. Let $G=(V, E)$ be a graph, where $V=V(G)$ is the set of vertices and $E=E(G)$ is the set of edges. We recall that a graph is connected if there exists a path connecting any two distinct vertices. The distance between two distinct vertices $x$ and $y$, denoted by $d(x, y)$, is the length of the shortest path connecting them (if such a path does not exist, then we set $d(x, y)=\infty)$. The diameter of a connected graph $\mathrm{G}$, denoted by $\operatorname{diam}(\mathrm{G})$, is the maximum distance between any pair of vertices of $G$. For a vertex $x$ in $G$, we denote the set of all vertices adjacent to $x$ by $N(x)$ and $N[x]=N(x) \cup\{x\}$. A k-partite graph is one whose vertex set can be partitioned into $k$ subsets so that an edge has both ends in no subset. A complete k-partite graph is a k-partite graph in which each vertex is adjacent to every vertex that is not in the same subset. The complete bipartite (i.e., 2-partite) graph with part sizes $m$ and $n$ is denoted by $K_{m, n}$. If $\mathrm{m}=1$, then the bipartite graph is called star. A graph in which each 
pair of vertices is joined by an edge is called a complete graph and use $\mathrm{K}_{\mathrm{n}}$ to denote it with $n$ vertices and its complement is denoted by $\bar{K}_{n}$ (possibly $n$ is zero). Also, a cycle of order $n$ is denoted by $C_{n}$. A subset of vertices $\mathrm{S} \subseteq \mathrm{V}(\mathrm{G})$ resolves a graph $\mathrm{G}$, and $\mathrm{S}$ is a resolving set of $\mathrm{G}$, if every vertex is uniquely determined by its vector of distances to the vertices of $S$. In general, for an ordered subset $S=\left\{v_{1}, v_{2}, \ldots, v_{k}\right\}$ of vertices in a connected graph $G$ and a vertex $v \in V(G) \backslash S$ of $G$, the metric representation of $v$ with respect to $S$ is the k-vector $D(v \mid S)=\left(d\left(v, v_{1}\right), d\left(v, v_{2}\right), \ldots, d\left(v, v_{k}\right)\right)$. The set $S$ is a resolving set for $G$ if $D(u \mid S)=D(v \mid S)$ implies that $u=v$, for all pair of vertices, $v, u \in V(G) \backslash S$. A resolving set $S$ of minimum cardinality is the metric basis for $\mathrm{G}$, and the number of elements in the resolving set of minimum cardinality is the metric dimension of $\mathrm{G}$. We denote the metric dimension of a graph $G$ by $\operatorname{dim}_{M}(G)$. Let $G$ be a connected graph such that $|V(G)| \geq 2$. Two distinct vertices $u$ and $v$ are distance similar, if $\mathrm{d}(\mathfrak{u}, \mathrm{x})=\mathrm{d}(\boldsymbol{v}, \mathrm{x})$, for all $x \in V(G) \backslash\{u, v\}$. It can be easily checked that two distinct vertices $u$ and $v$ are distance similar if either $u-v \notin E(G)$ and $N(u)=N(v)$ or $u-v \in E(G)$ and $\mathrm{N}[\mathrm{u}]=\mathrm{N}[\mathrm{v}]$.

Let $R$ be a commutative ring with identity and $A(R)$ be the set of ideals with non-zero annihilator. The strongly annihilating-ideal graph of $\mathrm{R}$ is defined as the graph $\operatorname{SAG}(R)$ with the vertex set $A(R)^{*}=A(R) \backslash\{0\}$ and two distinct vertices I and $J$ are adjacent if and only if $I \cap A n n(J) \neq(0)$ and $J \cap A n n(I) \neq(0)$. This graph was first introduced and studied in $[16,17]$. It is worthy to mention that strongly annihilating-ideal graph is a generalization of annihilating-ideal graph. The annihilating-ideal graph of $R$, denoted by $\mathbb{A} \mathbb{G}(R)$, is a graph with the vertex set $A(R)^{*}$ and two distinct vertices I and J are adjacent if and only if $\mathrm{IJ}=0$ (see [3] for more details). In this paper, we study the metric dimension of $\operatorname{SAG}(R)$ and we provide some metric dimension formulas for $S A G(R)$.

\section{Metric dimension of a strongly annihilating-ideal graph of a reduced ring}

Let $\mathrm{R}$ be a commutative ring. In this section, we provide a metric dimension formula for a strongly annihilating-ideal graph when $R$ is reduced.

Lemma 1 Let $\mathrm{R}$ be a ring which is not an integral domain. Then $\operatorname{dim}_{M}(\mathrm{SAG}(\mathrm{R}))$ is finite if and only if $\mathrm{R}$ has only finitely many ideals.

Proof. One side is clear. To prove the other side, suppose that $\operatorname{dim}_{M}(\operatorname{SAG}(R))$ is finite and let $W=\left\{I_{1}, I_{2}, \ldots, I_{n}\right\}$ be the metric basis for $\operatorname{SAG}(R)$, where $n$ 
is a non-negative. By [16, Theorem 2.1], $\operatorname{diam}(\operatorname{SAG}(\mathrm{R})) \leq 2$ and so for every $I \in A(R)^{*} \backslash W$, there are $(2+1)^{n}$ possibilities for $D(I \mid W)$. Thus $\left|A(R)^{*}\right| \leq 3^{n}+n$ and hence $R$ has only finitely many ideals.

If $R$ is a reduced ring with finitely many ideals, then by [2, Theorem 8.7], $R$ is a direct product of finitely many fields. Using this fact, we prove the following result.

Theorem 1 Let $\mathrm{R}$ be a reduced ring which is not an integral domain. If $\operatorname{dim}_{M}(\operatorname{SAG}(\mathrm{R}))$ is finite, then:

(1) If $|\operatorname{Max}(R)| \leq 3$, then $\operatorname{dim}_{M}(\operatorname{SAG}(R))=|\operatorname{Max}(R)|-1$.

(2) If $|\operatorname{Max}(R)| \geq 4$, then $\operatorname{dim}_{M}(\operatorname{SAG}(R))=|\operatorname{Max}(R)|$.

Proof. (1) Since $\operatorname{dim}_{M}(\operatorname{SAG}(R))$ is finite, $R$ has only finitely many ideals, by Lemma 1 . Also, since $R$ is not an integral domain, $|\operatorname{Max}(R)| \neq 1$. Hence $|\operatorname{Max}(R)|=2$ or 3. If $|\operatorname{Max}(R)|=2$, then $R \cong F_{1} \times F_{2}$, where $F_{i}$ is a field. Thus $\operatorname{SAG}(R)=K_{2}$ and so $\operatorname{dim}_{M}(\operatorname{SAG}(R))=1$. If $|\operatorname{Max}(R)|=3$, then $R \cong F_{1} \times F_{2} \times$ $F_{3}$, where $F_{i}$ is a field for every $1 \leq i \leq 3$. Let $W=\left\{F_{1} \times(0) \times F_{3}, F_{1} \times F_{2} \times(0)\right\}$. By the following figure, one may easily get
$\mathrm{D}\left((0) \times \mathrm{F}_{2} \times(0) \mid \mathrm{W}\right)=(1,2)$,
$\mathrm{D}\left(\mathrm{F}_{1} \times(0) \times(0) \mid \mathrm{W}\right)=(2,2)$,
$\mathrm{D}\left((0) \times(0) \times \mathrm{F}_{3} \mid \mathrm{W}\right)=(2,1)$,
$\mathrm{D}\left((0) \times \mathrm{F}_{2} \times \mathrm{F}_{3} \mid \mathrm{W}\right)=(1,1)$.

So for every $x, y \in V(S A G(R)) \backslash W, D(x \mid W) \neq D(y \mid W)$ and hence $\operatorname{dim}_{M}(S A G$ $(R))=2$.

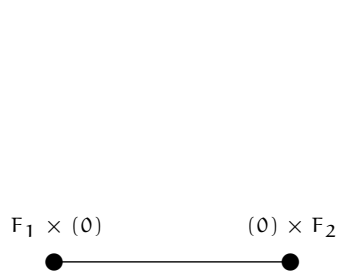

$\operatorname{SAG}\left(F_{1} \times F_{2}\right)$

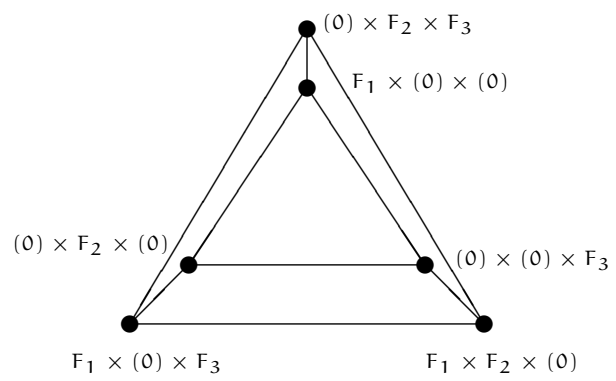

$\operatorname{SAG}\left(F_{1} \times F_{2} \times F_{3}\right)$

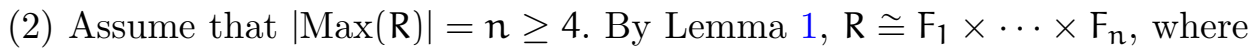


$F_{i}$ is a field for every $1 \leq i \leq n$. We show that $\operatorname{dim}_{M}(\operatorname{SAG}(R))=n$. Indeed, we have the following claims:

Claim 1. $\operatorname{dim}_{M}(\operatorname{SAG}(R)) \geq n$.

Since $R \cong F_{1} \times \cdots \times F_{n}$, by Lemma $1, \operatorname{dim}_{M}(\operatorname{SAG}(R))$ is finite. Let $W=$ $\left\{I_{1}, I_{2}, \ldots, I_{k}\right\}$ be the metric basis for $\operatorname{SAG}(R)$, where $k$ is a positive integer. On the other hand, by [16, Theorem 2.1], diam(SAG(R)) $\in\{1,2\}$, and so for every $I \in A(R)^{*} \backslash W$, there are $2^{k}$ possibilities for $D(I \mid W)$. This implies that $\left|A(R)^{*}\right|-k \leq 2^{k}$. Since $\left|A(R)^{*}\right|=2^{n}-2,2^{n}-2-k \leq 2^{k}$ and hence $2^{n} \leq 2^{k}+2+k$. Since $n \geq 4$, we deduce that $k \geq n$. Therefore $\operatorname{dim}_{M}(\operatorname{SAG}(R)) \geq n$.

Claim 2. $\operatorname{dim}_{M}(S A G(R)) \leq n$.

For every $1 \leq i \leq n$, let $\left(F_{1}, \ldots, F_{i-1}, 0, F_{i+1}, \ldots, F_{n}\right)=\mathfrak{m}_{i} \in A(R)^{*}$. Put $W=\left\{\mathfrak{m}_{1}, \mathfrak{m}_{2}, \ldots, \mathfrak{m}_{n}\right\}$ (in fact $\left.W=\operatorname{Max}(R)\right)$. We show that $W$ is the resolving set for $\operatorname{SAG}(R)$. To see this, let $I, J \in V(S A G(R)) \backslash W$ and $I \neq J$. We need only to show that $\mathrm{D}(\mathrm{I} \mid \mathrm{W}) \neq \mathrm{D}(\mathrm{J} \mid \mathrm{W})$. Let $\mathrm{I}=\left(\mathrm{I}_{1}, \mathrm{I}_{2}, \ldots, \mathrm{I}_{\mathrm{n}}\right)$ and $\mathrm{J}=\left(\mathrm{J}_{1}, \mathrm{~J}_{2}, \ldots, \mathrm{J}_{\mathrm{n}}\right)$. Since $I \neq J, I_{i}=0$ and $J_{i}=F_{i}$ or $I_{i}=F_{i}$ and $J_{i}=0$, for some $1 \leq i \leq n$. Without loss of generality, assume that $I_{1}=0$ and $J_{1}=F_{1}$. It is easy to see that $d\left(I, \mathfrak{m}_{1}\right)=1$ and $d\left(J, \mathfrak{m}_{1}\right)=2$. This clearly shows that $D(I \mid W) \neq D(J \mid W)$. Therefore $\operatorname{dim}_{M}(\operatorname{SAG}(\mathrm{R})) \leq \mathrm{n}$.

Now, by Claims 1, 2, $\operatorname{dim}_{M}(\operatorname{SAG}(R))=\mathfrak{n}$, for $n \geq 4$.

\section{Metric dimension of a strongly annihilating-ideal graph of a non-reduced ring}

In this section, we discuss the metric dimension of strongly annihilating-ideal graphs for non-reduced rings. First we need to recall two lemmas from [16].

Lemma 2 [16, Lemma 2.1] Let $\mathrm{R}$ be a ring and $\mathrm{I}, \mathrm{J} \in \mathrm{A}(\mathrm{R})^{*}$. Then the following statements hold.

(1) If $\mathrm{I}-\mathrm{J}$ is not an edge of $\mathrm{SAG}(\mathrm{R})$, then $\mathrm{Ann}(\mathrm{IJ})=\operatorname{Ann}(\mathrm{I})$ or $\mathrm{Ann}(\mathrm{IJ})=$ $\mathrm{Ann}(\mathrm{J})$. Moreover, if $\mathrm{R}$ is a reduced ring, then the converse is also true.

(2) If $\mathrm{I}-\mathrm{J}$ is an edge of $\mathbb{A} G(R)$, then $\mathrm{I}-\mathrm{J}$ is an edge of $\mathrm{SAG}(\mathrm{R})$.

(3) If $\mathrm{Ann}(\mathrm{I}) \nsubseteq \mathrm{Ann}(\mathrm{J})$ and $\mathrm{Ann}(\mathrm{J}) \nsubseteq \mathrm{Ann}(\mathrm{I})$, then $\mathrm{I}-\mathrm{J}$ is an edge of $\mathrm{SAG}(\mathrm{R})$. Moreover if $\mathrm{R}$ is a reduced ring, then the converse is also true. 
(4) Let $\mathrm{n} \geq 1$ be a positive integer. Suppose that $\mathrm{R} \cong \mathrm{R}_{1} \times \cdots \times \mathrm{R}_{\mathrm{n}}$, where $\mathrm{R}_{\mathrm{i}}$ is a ring, for every $1 \leq \mathrm{i} \leq \mathrm{n}$, and $\mathrm{I}=\left(\mathrm{I}_{1}, \ldots, \mathrm{I}_{\mathrm{n}}\right)$ and $\mathrm{J}=\left(\mathrm{J}_{1}, \ldots, \mathrm{J}_{\mathrm{n}}\right)$ are two vertices of $\mathrm{SAG}(\mathrm{R})$. If $\mathrm{I}_{i} \cap \operatorname{Ann}\left(\mathrm{J}_{i}\right) \neq(0)$ and $\mathrm{J}_{j} \cap \operatorname{Ann}\left(\mathrm{I}_{j}\right) \neq(0)$, for some $1 \leq \mathrm{i}, \mathrm{j} \leq \mathrm{n}$, then $\mathrm{I}-\mathrm{J}$ is an edge of $\mathrm{SAG}(\mathrm{R})$. In particular, if $\mathrm{I}_{i}-\mathrm{J}_{i}$ is an edge of $\mathrm{SAG}\left(\mathrm{R}_{i}\right)$ or $\mathrm{I}_{i}=\mathrm{J}_{i}$ and $\mathrm{I}_{i} \cap \operatorname{Ann}\left(\mathrm{I}_{i}\right) \neq(0)$, for some $1 \leq \mathrm{i} \leq \mathrm{n}$, then $\mathrm{I}-\mathrm{J}$ is an edge of $\mathrm{SAG}(\mathrm{R})$.

(5) If $\mathrm{I}, \mathrm{J} \in \operatorname{Ess}(\mathrm{R})$ or $\operatorname{Ann}(\mathrm{I}), \operatorname{Ann}(\mathrm{J}) \in \operatorname{Ess}(\mathrm{R})$, then $\mathrm{I}$ is adjacent to $\mathrm{J}$.

(6) If $\mathrm{d}_{\mathbb{A G}(\mathrm{R})}(\mathrm{I}, \mathrm{J})=3$ for some distinct $\mathrm{I}, \mathrm{J} \in \mathrm{A}(\mathrm{R})^{*}$, then $\mathrm{I}-\mathrm{J}$ is an edge of $\operatorname{SAG}(\mathrm{R})$.

(7) If $\mathrm{I}-\mathrm{J}$ is not an edge of $\mathrm{SAG}(\mathrm{R})$ for some distinct $\mathrm{I}, \mathrm{J} \in \mathrm{A}(\mathrm{R})^{*}$, then $\mathrm{d}_{\mathbb{A} \mathbb{G}(R)}(\mathrm{I}, \mathrm{J})=2$.

Lemma 3 [16, Lemma 2.2] Let $\mathrm{R}$ be a non-reduced ring and $\mathrm{I}$ be an ideal of $\mathrm{R}$ such that $\mathrm{I}^{\mathrm{n}}=(0)$, for some positive integer $\mathrm{n}$. Then $\mathrm{Ann}(\mathrm{I})$ is an essential ideal of $\mathrm{R}$.

Remark 1 Let $\mathrm{G}$ be a connected graph and $\mathrm{V}_{1}, \mathrm{~V}_{2}, \ldots, \mathrm{V}_{\mathrm{k}}$ be a partition of $\mathrm{V}(\mathrm{G})$ such that for every $1 \leq \mathrm{i} \leq \mathrm{k}$, if $\mathrm{x}, \mathrm{y} \in \mathrm{V}_{\mathrm{i}}$, then $\mathrm{N}(\mathrm{x})=\mathrm{N}(\mathrm{y})$. Then $\operatorname{dim}_{M}(G) \geq|V(G)|-k$.

Next, we provide some formulas for the metric dimension of strongly annihilatingideal graphs for non-reduced rings.

Theorem 2 Suppose that $\mathrm{R} \cong \mathrm{R}_{1} \times \cdots \times \mathrm{R}_{\mathrm{n}}$, where $\mathrm{R}_{\mathrm{i}}$ is an Artinian local ring such that for every $1 \leq i \leq n,\left|A\left(R_{i}\right)^{*}\right|=1$. Then $\operatorname{dim}_{M}(\operatorname{SAG}(R))=2 n$.

Proof. Assume that $X=\left(R_{1}, 0, \ldots, 0\right)$ and $Y=\left(I_{1}, 0, \ldots, 0\right)$, where $I_{1} \in$ $A\left(R_{1}\right)^{*}$. By Part 4 of Lemma 2, it is easy to see that $N(X)=N(Y)$. This implies that if $W$ is the metric basis for $\operatorname{SAG}(R)$, then $X \in W$ or $Y \in W$. Without loss of generality, we may assume that $X \in W$. Similarly, we may assume that $W_{1} \subseteq W$, where $W_{1}=\left\{\left(R_{1}, 0, \ldots, 0\right),\left(0, R_{2}, 0, \ldots, 0\right), \ldots,\left(0, \ldots, 0, R_{n}\right)\right\}$.

Now, assume that $X=\left(0, R_{2}, \ldots, R_{n}\right)$ and $Y=\left(I_{1}, R_{2}, \ldots, R_{n}\right)$, where $I_{1} \in$ $A\left(R_{1}\right)^{*}$. It is easy to see that $N(X)=N(Y)$ and so if $W$ is the metric basis for $\operatorname{SAG}(R)$, then $X \in W$ or $Y \in W$. Without loss of generality, we may assume that $X \in W$. Similarly, we may assume that $W_{2} \subseteq W$, where

$$
W_{2}=\left\{\left(0, R_{2}, \ldots, R_{n}\right),\left(R_{1}, 0, R_{3}, \ldots, R_{n}\right), \ldots,\left(R_{1}, \ldots, R_{n-1}, 0\right)\right\} .
$$

Since $\left|W_{1}\right|=\left|W_{2}\right|=n$ and $W_{1} \cup W_{2} \subseteq W,|W| \geq 2 n$. We show that $|W| \leq 2 n$. For this, it is enough to show that $W$ is a resolving set and consequently it is 
the metric basis for the graph $\mathrm{SAG}(\mathrm{R})$. Let $X, Y \notin W, X \neq Y, X=\left(I_{1}, \ldots, I_{n}\right)$ and $Y=\left(J_{1}, \ldots, J_{n}\right)$. We show that $D(X \mid W) \neq D(Y \mid W)$. Since $X \neq Y$, for some $1 \leq i \leq n$, we conclude that $\mathrm{I}_{i} \neq \mathrm{J}_{i}$. Without loss of generality, one may assume that $I_{1} \supset J_{1}$. We have the following cases:

Case 1. $I_{1}=R_{1}$.

Subcase 1. For some $2 \leq j \leq n, J_{j} \neq 0$. In this case, $Z-Y$ is an edge of $\operatorname{SAG}(R)$ but $Z-X$ is not an edge of $\operatorname{SAG}(R)$, where $Z=\left(R_{1}, 0, \ldots, 0\right)$. Since $Z \in W$, we deduce that $D(X \mid W) \neq D(Y \mid W)$.

Subcase 2. For every $2 \leq j \leq n, J_{j}=0$. Since $I_{1}=R_{1}$ and $\left(R_{1}, 0, \ldots, 0\right) \in W$, for some $2 \leq i \leq n, I_{i} \neq 0$. If $I_{i}=R_{i}$, for some $2 \leq i \leq n$, then $Z-Y$ is an edge of $\operatorname{SAG}(R)$ but $Z-X$ is not an edge of $\operatorname{SAG}(R)$, where $Z=\left(0, \ldots, 0, R_{i}, 0 \ldots, 0\right)$. So we can let for every $2 \leq i \leq n, I_{i} \neq R_{i}$. Now, without loss of generality, we may assume that $I_{2} \neq 0$. Obviously, $Z-X$ is an edge of $S A G(R)$ but $Z-Y$ is not an edge of $\operatorname{SAG}(R)$, where $Z=\left(R_{1}, 0, R_{3} \ldots, R_{n}\right)$. Since $Z \in W$, $\mathrm{D}(\mathrm{X} \mid \mathrm{W}) \neq \mathrm{D}(\mathrm{Y} \mid \mathrm{W})$.

Case 2. $I_{1} \neq R_{1}$. Since $I_{1} \neq R_{1}, J_{1} \neq R_{1}$. Also, since $X \neq Y$, we may let $I_{1} \in A\left(R_{1}\right)^{*}$ and $J_{1}=0$. If $I_{i} \neq R_{i}$, for some $2 \leq i \leq n$, then $Z-X$ is an edge of $\operatorname{SAG}(R)$ but $Z-Y$ is not an edge of $S A G(R)$, where $Z=\left(0, R_{2}, R_{3} \ldots, R_{n}\right)$. Since $Z \in W, D(X \mid W) \neq D(Y \mid W)$. So let $X=\left(I_{1}, R_{2}, \ldots, R_{n}\right)$. Since $J_{1}=0$ and $Y \notin W$, for some $2 \leq i \leq n, J_{i} \in A\left(R_{1}\right)^{*}$. Without loss of generality, we may assume that $J_{2} \in A\left(R_{2}\right)^{*}$. If $J_{i} \neq 0$, for some $3 \leq i \leq n$, then we put $Z=\left(0, R_{2}, \ldots, R_{i-1}, 0, R_{i+1}, \ldots, R_{n}\right)$. It is not hard to check that $Z-Y$ is an edge of $\operatorname{SAG}(R)$ but $Z-X$ is not an edge of $\operatorname{SAG}(R)$. If for every $3 \leq i \leq n$, $J_{i}=0$, then we put $Z=\left(R_{1}, R_{2}, \ldots, 0, \ldots, 0\right)$. In both cases we have that $\mathrm{D}(\mathrm{X} \mid \mathrm{W}) \neq \mathrm{D}(\mathrm{Y} \mid \mathrm{W})$. Therefore, $|\mathrm{W}| \leq 2 \mathrm{n}$.

Theorem 3 Suppose that $\mathrm{R} \cong \mathrm{R}_{1} \times \cdots \times \mathrm{R}_{\mathrm{n}}$, where $\mathrm{R}_{\mathrm{i}}$ is an Artinian local ring such that for every $1 \leq i \leq n,\left|A\left(R_{i}\right)^{*}\right| \geq 2$. Then $\operatorname{dim}_{M}(\operatorname{SAG}(R))=$ $\left|A(R)^{*}\right|-3^{n}+2$.

Proof. If $R$ is local, then Lemma 3 implies that $S A G(R)$ is complete and hence $\operatorname{dim}_{M}(\operatorname{SAG}(R))=\left|A(R)^{*}\right|-1$. So let $R \cong R_{1} \times \cdots \times R_{n}$ and $n \geq 2$. Assume that

$X=\left(I_{1}, \ldots, I_{n}\right), Y=\left(J_{1}, \ldots, J_{n}\right)$ are vertices of $\operatorname{SAG}(R)$. Define the relation $\sim$ on $\mathrm{V}(\mathrm{SAG}(\mathrm{R}))$ as follows: $\mathrm{X} \sim \mathrm{Y}$, whenever, the following two conditions hold.

(1) " $I_{i}=0$ if and only if $J_{i}=0$ " for every $1 \leq i \leq n$. 
(2) " $0 \neq \mathrm{I}_{i} \subseteq \operatorname{Nil}\left(R_{i}\right)$ if and only if $0 \neq \mathrm{J}_{i} \subseteq \operatorname{Nil}\left(R_{i}\right)$ " for every $1 \leq i \leq n$.

It is easily seen that $\sim$ is an equivalence relation on $V(\operatorname{SAG}(R))$. By $[X]$, we mean the equivalence class of $X$. Let $X_{1}$ and $X_{2}$ be two elements of $[X]$. Since $X_{1} \sim X_{2}$, by Part 4 of Lemma $2, N\left(X_{1}\right)=N\left(X_{2}\right)$. This, together with the fact that the number of equivalence classes is $3^{n}-2$ and Remark 1 , implies that

$$
\operatorname{dim}_{M}(\operatorname{SAG}(R)) \geq\left|A(R)^{*}\right|-\left(3^{n}-2\right)=\left|A(R)^{*}\right|-3^{n}+2 .
$$

We show that

$$
\operatorname{dim}_{M}(\operatorname{SAG}(R)) \leq\left|A(R)^{*}\right|-3^{n}+2
$$

Let

$A=\left\{\left(I_{1}, \ldots, I_{n}\right) \in V(\operatorname{SAG}(R)) \mid I_{i} \in\left\{0, \operatorname{Nil}\left(R_{i}\right), \ldots, R_{i}\right\}\right.$ for every $\left.1 \leq i \leq n\right\}$ and $W=A(R)^{*} \backslash A$.

It is shown that $W$ is a resolving set and consequently it is the metric basis for the graph $\operatorname{SAG}(R)$. To see this, let $X, Y \in A$ and $X \neq Y$. We show that $D(X \mid W) \neq D(Y \mid W)$. Let $X=\left(I_{1}, \ldots, I_{n}\right)$ and $Y=\left(J_{1}, \ldots, J_{n}\right)$. Since $X \neq Y$, for some $1 \leq i \leq n, I_{i} \neq J_{i}$. Without loss of generality, we may assume that $\mathrm{I}_{1} \supset \mathrm{J}_{1}$. We have the following cases:

Case 1. $I_{1}=R_{1}$.

Subcase 1. $J_{1}=0$. In this case $Z-X$ is an edge of $S A G(R)$ but $Z-Y$ is not an edge of $\operatorname{SAG}(R)$, where $Z=\left(I_{1}^{\prime}, R_{2}, \ldots, R_{n}\right)$ and $I_{1}^{\prime} \in A\left(R_{1}\right)^{*} \backslash\left\{\operatorname{Nil}\left(R_{1}\right)\right\}$. Since $Z \in W, D(X \mid W) \neq D(Y \mid W)$. Subcase 2. $J_{1}=\operatorname{Nil}\left(R_{1}\right)$. In this case $Z-Y$ is an edge of $\operatorname{SAG}(R)$ but $Z-X$ is not an edge of $\operatorname{SAG}(R)$, where $Z=\left(J_{1}^{\prime}, 0, \ldots, 0\right)$, $J_{1}^{\prime} \in A\left(R_{1}\right)^{*}$ and $J_{1}^{\prime} \neq \operatorname{Nil}\left(R_{1}\right)$. Since $Z \in W, D(X \mid W) \neq D(Y \mid W)$.

Case 2. $I_{1}=\operatorname{Nil}\left(R_{1}\right)$.

Since $I_{1} \neq J_{1}$ and $I_{1} \supseteq J_{1}, J_{1}=0$. Hence $Z-X$ is an edge of $\operatorname{SAG}(R)$ but $Z-Y$ is not an edge of $\operatorname{SAG}(R)$, where $Z=\left(J_{1}^{\prime}, R_{2}, \ldots, R_{n}\right)$ and $J_{1}^{\prime} \in A\left(R_{1}\right)^{*}$ and $J_{1}^{\prime} \neq \operatorname{Nil}\left(R_{1}\right)$. Since $Z \in W, D(X \mid W) \neq D(Y \mid W)$. Therefore,

$$
\operatorname{dim}_{M}(\operatorname{SAG}(R)) \leq|W| \text {. }
$$

Since $|A|=3^{n}-2,|W|=\left|A(R)^{*}\right|-\left(3^{n}-2\right)=\left|A(R)^{*}\right|-3^{n}+2$. Therefore,

$$
\operatorname{dim}_{M}(\operatorname{SAG}(R)) \leq\left|A(R)^{*}\right|-3^{n}+2 .
$$

Next, we provide some upper and lower bounds for the metric dimension of strongly annihilating-ideal graphs for some other classes of non-reduced rings. 
Theorem 4 Suppose that $\mathrm{R} \cong \mathrm{R}_{1} \times \cdots \times \mathrm{R}_{\mathrm{n}} \times \mathrm{F}_{\mathrm{n}+1} \times \cdots \times \mathrm{F}_{\mathrm{n}+\mathrm{m}}$, where $\mathrm{R}_{\mathrm{i}}$ is an Artinian local ring such that $\left|A\left(R_{i}\right)\right|=2$ for every $1 \leq i \leq n$ and $F_{i}$ is a field for every $1+n \leq i \leq n+m$. Then $n+m \leq \operatorname{dim}_{M}(\operatorname{SAG}(R)) \leq 2^{n+m}-2$.

Proof. Suppose that $W=\left\{I_{1}, I_{2}, \ldots, I_{k}\right\}$ be the metric basis for $\operatorname{SAG}(R)$, for some non-negative integer $k$. Since $\operatorname{diam}(\operatorname{SAG}(R)) \leq 2$, there are exactly $(2)^{k}$ possibilities for $D(I \mid W)$, for every $I \in A(R)^{*} \backslash W$. On the other hand, since $\left|A(R)^{*}\right|=3^{n} 2^{m}-2$, we must have $3^{n} 2^{m}-2-k \leq 2^{k}$. This implies that $n+m \leq k$. Hence $n+m \leq \operatorname{dim}_{M}(\operatorname{SAG}(R))$. It is shown that $\operatorname{dim}_{M}(\operatorname{SAG}(R)) \leq$ $2^{\mathrm{n}+\mathrm{m}}-2$. Let

$W=\left\{\left(I_{1}, \ldots, I_{n+m}\right) \in V(S A G(R)) \mid I_{i} \in\left\{0, R_{1}, \ldots, R_{n}, F_{1}, \ldots, F_{m}\right\}\right.$ for every $1 \leq i \leq n+m\}$.

We show that $W$ is a resolving set for $S A G(R)$. For this, let $X, Y \in A(R)^{*} \backslash W$ and $X \neq Y$. We show that $D(X \mid W) \neq D(Y \mid W)$. Let $X=\left(I_{1}, \ldots, I_{n+m}\right)$ and $Y=\left(J_{1}, \ldots, J_{n+m}\right)$. Since $X \neq Y, I_{i} \neq J_{i}$, for some $1 \leq i \leq n+m$.

We have the following cases:

Case 1. For some $n+1 \leq i \leq n+m, I_{i} \neq J_{i}$.

Without loss of generality, we may assume that $i=n+m, I_{n+m}=F_{n+m}$ and $J_{n+m}=0$. Now, put $Z=\left(R_{1}, \ldots, R_{n}, F_{n+1} \ldots, F_{n+m-1}, 0\right)$. Since for some $1 \leq i \leq n, I_{i} \in A\left(R_{i}\right)^{*}$, one may easily see that $Z-X$ is an edge of $\operatorname{SAG}(R)$ but $Z-Y$ is not an edge of $S A G(R)$. Since $Z \in W, D(X \mid W) \neq D(Y \mid W)$.

Case 2. For every $n+1 \leq i \leq n+m, I_{i}=J_{i}$.

Since $I_{i} \neq J_{i}$, for some $1 \leq i \leq n$, one can let $J_{1} \subset I_{1}$. Thus we have the following subcases:

Subcase 1. $J_{1}=0$ and $I_{1} \in A\left(R_{1}\right)^{*}$.

Since $J_{1}=0$, for some $2 \leq i \leq n, J_{i} \in A\left(R_{i}\right)^{*}$. Hence one can let $J_{2} \in A\left(R_{2}\right)^{*}$. If for some $2 \leq i \leq n, I_{i} \neq R_{i}$ or for some $1+m \leq i \leq n+m, I_{i} \neq F_{i}$, then put $Z=\left(0, R_{2}, R_{3} \ldots, R_{n}, F_{n+1}, \ldots, F_{n+m}\right) . Z-X$ is an edge of $\operatorname{SAG}(R)$ but $Z-Y$ is not an edge of $\operatorname{SAG}(R)$. Since $Z \in W, D(X \mid W) \neq D(Y \mid W)$. So we let $X=\left(I_{1}, R_{2} \ldots, R_{n}, F_{n+1}, \ldots, F_{n+m}\right)$ Similarly, if for some $3 \leq i \leq n, J_{i} \neq R_{i}$ or for some $1+m \leq j \leq n+m, J_{i} \neq F_{i}$, then without loss of generality, we may assume that $J_{3} \neq R_{3}$. Then put $Z=\left(0,0, R_{3} \ldots, R_{n}, F_{n+1}, \ldots, F_{n+m}\right)$. Thus $Z-Y$ is an edge of $\operatorname{SAG}(R)$ but $Z-X$ is not an edge of $\operatorname{SAG}(R)$. Since $Z \in W, D(X \mid W) \neq D(Y \mid W)$. Now, let $X=\left(I_{1}, R_{2} \ldots, R_{n}, F_{n+1}, \ldots, F_{n+m}\right)$ and $Y=\left(0, J_{2}, R_{3}, \ldots, R_{n}, F_{n+1}, \ldots, F_{n+m}\right)$. Put $Z=\left(0, R_{2}, 0 \ldots, 0,0, \ldots, 0\right)$. Therefore, $Z-Y$ is an edge of $\operatorname{SAG}(R)$ but $Z-X$ is not an edge of $\operatorname{SAG}(R)$. Since $Z \in W, D(X \mid W) \neq D(Y \mid W)$. 
Subcase 2. $\mathrm{J}_{1}=0$ and $\mathrm{I}_{1}=\mathrm{R}_{1}$.

Since $J_{1}=0$, for some $2 \leq i \leq n, J_{i} \in A\left(R_{i}\right)^{*}$. Hence one may let $J_{2} \in A\left(R_{2}\right)^{*}$. Assume that $Z=\left(R_{1}, 0, \ldots, 0\right)$. Thus $Z-Y$ is an edge of $S A G(R)$ but $Z-X$ is not an edge of $\operatorname{SAG}(R)$ (note that since $Z \in W, Z \neq X$ ). This implies that $\mathrm{D}(\mathrm{X} \mid \mathrm{W}) \neq \mathrm{D}(\mathrm{Y} \mid \mathrm{W})$.

Subcase 3. $J_{1} \in A\left(R_{1}\right)^{*}$ and $I_{1}=R_{1}$. If $J_{i} \neq 0$, for some $2 \leq i \leq n$, then one may assume that $J_{2} \neq 0$. Suppose that $Z=\left(R_{1}, 0, \ldots, 0\right)$. Then $Z-Y$ is an edge of $\operatorname{SAG}(R)$ but $Z-X$ is not an edge of $\operatorname{SAG}(R)$. Hence $D(X \mid W) \neq D(Y \mid W)$. Let $Y=\left(J_{1}, 0, \ldots, 0\right)$. Since $X \notin W$, for some $2 \leq i \leq n, I_{i} \in A\left(R_{i}\right)^{*}$. So, we can let $I_{2} \in A\left(R_{2}\right)^{*}$. If $I_{i} \neq 0$, for some $3 \leq i \leq n$, then we can assume that $I_{3} \neq 0$. If we put $Z=\left(R_{1}, R_{2}, 0, \ldots, 0\right)$, then we easily get $D(X \mid W) \neq D(Y \mid W)$. Finally, if $X=\left(R_{1}, I_{2}, 0, \ldots, 0\right)$ and $Y=\left(J_{1}, 0, \ldots, 0\right)$, then $D(X \mid W) \neq D(Y \mid W)$. Since $Z-X$ is an edge of $S A G(R)$ but $Z-Y$ is not an edge of $\operatorname{SAG}(R)$, where $Z=$ $\left(R_{1}, 0, R_{3}, 0 \ldots, 0\right)$. Therefore, $\operatorname{dim}_{M}(\operatorname{SAG}(R)) \leq|W|$. Since $|W|=2^{n+m}-2$, $\operatorname{dim}_{M}(\operatorname{SAG}(R)) \leq 2^{\mathrm{n}+\mathrm{m}}-2$.

We end this paper with the following example.

Example 1 (1) Let $\mathrm{R}=\mathbb{Z}_{4} \times \mathbb{Z}_{2}$. Then $\mathrm{SAG}(\mathrm{R})=\mathrm{C}_{4}$ and hence $\operatorname{dim}_{\mathrm{M}}(\mathrm{SAG}$ $(\mathrm{R}))=2$. Also, in Theorem $4, \mathrm{n}=\mathrm{m}=1$, and so $\operatorname{dim}_{M}(\operatorname{SAG}(\mathrm{R}))=2$.

(2) Let $\mathrm{R}=\mathbb{Z}_{4} \times \mathbb{Z}_{2} \times \mathbb{Z}_{2}$ and $\operatorname{dim}_{M}(\operatorname{SAG}(\mathrm{R}))=\mathrm{k}$. We show that $3 \leq \mathrm{k} \leq 6$. Since $\operatorname{diam}(\operatorname{SAG}(R)) \leq 2$ and $\left|A(R)^{*}\right|=10,10-k \leq 2^{k}$. Thus $k \geq 3$. Let $W=\left\{\left((2), \mathbb{Z}_{2}, \mathbb{Z}_{2}\right),\left((2), 0, \mathbb{Z}_{2}\right),\left((2), \mathbb{Z}_{2}, 0\right),((2), 0,0)\right\}$. Then

$\mathrm{D}\left(\left(\mathbb{Z}_{4}, 0,0\right) \mid \mathrm{W}\right)=(1,1,1,2)$,

$\left.\mathrm{D}\left(\left(\mathbb{Z}_{4}, \mathbb{Z}_{2}, 0\right)\right) \mid \mathrm{W}\right)=(1,1,2,2)$,

$\left.\mathrm{D}\left(\left(\mathbb{Z}_{4}, 0, \mathbb{Z}_{2}\right)\right) \mid \mathrm{W}\right)=(1,2,1,2)$,

$\mathrm{D}\left(\left(0, \mathbb{Z}_{2}, \mathbb{Z}_{2}\right) \mid \mathrm{W}\right)=(2,1,1,1)$,

$\mathrm{D}\left(\left(0, \mathbb{Z}_{2}, 0\right) \mid \mathrm{W}\right)=(2,1,2,1)$,

$\mathrm{D}\left(\left(0,0, \mathbb{Z}_{2}\right) \mid \mathrm{W}\right)=(2,2,1,1)$.

Therefore, $\mathrm{W}$ is a resolving set for $\mathrm{SAG}(\mathrm{R})$ and hence $\mathrm{k} \leq 6$.

\section{Acknowledgements}

The authors express their deep gratitude to the referees for their valuable suggestions which have definitely improved the paper. 


\section{References}

[1] F. Ali, M. Salman, S. Huang, On the commuting graph of dihedral group, Comm. Algebra., 44 (2016), 2389-2401.

[2] M. F. Atiyah, I. G. Macdonald, Introduction to Commutative Algebra, Addison-Wesley Publishing Company, (1969).

[3] M. Behboodi, The annihilating-ideal graph of a commutative ring I, $J$. Algebra Appl., 10 (2011), 727-739.

[4] W. Bruns and J. Herzog, Cohen-Macaulay Rings, Cambridge University Press (1997).

[5] G. Chartrand, L. Eroh, M. A. Johnson, O. R. Oellermann, Resolvability in graphs and the metric dimension of a graph, Disc. Appl. Math., 105 (2000), 99-113.

[6] D. Dolžan, The metric dimension of the total graph of a finite commutative ring, Canad. Math. Bull., 59 (2016), 748-759.

[7] F. Harary and R. A. Melter, On the metric domension of a graph, Ars Combin., 2 (1976), 191-195.

[8] S. Khuller, B. Raghavachari, A. Rosenfeld, Localization in graphs, Technical report CS-TR-3326, University of Maryland at College Park, 1994.

[9] S. Pirzada, R. Raja and S. P. Redmond, Locating sets and numbers of graphs associated to commutative rings, J. Algebra Appl. 13:7 (2014): 145004718 pp.

[10] S. Pirzada, R. Raja, On the metric domension of a zero-divisor graph, Communications in Algebra, 45:4 (2017), 1399-1408.

[11] S. Pirzada, Rameez Raja, On graphs associated with modules over commutative rings, J. Korean. Math. Soc., 53 (2016), 1167-1182.

[12] R. Raja, S. Pirzada and S. P. Redmond, On Locating numbers and codes of zero-divisor graphs associated with commutative rings, J. Algebra Appl., 15:1 (2016): 165001422 pp.

[13] S. Pirzada, M. Imran Bhat, Computing metric dimension of compressed zero divisor graphs associated to rings, Acta Univ. Sapientiae, Mathematica, 10 (2) (2018), 298-318. 
[14] A. Sebö, E. Tannier, On metric generators of graphs, Math. Oper. Res., 29 (2004), 383-393.

[15] V. Soleymanivarniab, A. Tehranian, R. Nikandish, The metric dimension of annihilator graphs of commtative rings, J. Algebra Appl., to appear.

[16] N. KH. Tohidi, M. J. Nikmehr, R. Nikandish, On the strongly annihilating-ideal graph of a commutative ring, Discrete Math. Algorithm. Appl., 09, 1750028 (2017) [13 pages].

[17] N. KH. Tohidi, M. J. Nikmehr, R. Nikandish, Some results on the strongly annihilating-ideal graph of a commutative ring, Bol. Soc. Mat. Mex., 24, (2018), 307-318.

[18] D. B. West, Introduction to Graph Theory, $2^{\text {nd }}$ ed., Prentice Hall, Upper Saddle River (2001).

Received: February 23, 2020 